\title{
Detection Methods and Status of CAT Interruption of ATXN1 in Korean Patients With Spinocerebellar Ataxia Type 1
}

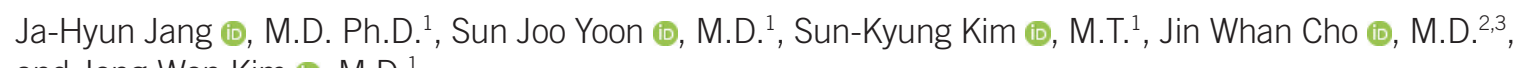
and Jong-Won Kim (iD, M.D. ${ }^{1}$

Departments of ${ }^{1}$ Laboratory Medicine and Genetics and ${ }^{2}$ Neurology, Samsung Medical Center, Sungkyunkwan University School of Medicine, Seoul, Korea; ${ }^{3}$ Neuroscience Center, Samsung Medical Center, Seoul, Korea

Spinocerebellar ataxia type 1 (SCA1) is an autosomal dominant disease caused by abnormal CAG repeat expansion in the ataxin 1 gene (ATXN1). The presence of CAT interruption(s) is important for diagnosing SCA1 in patients with 39-44 repeat alleles, as only uninterrupted alleles are considered abnormal. Determining the CAT interruption status might also be important for patients with $>44$ repeats, as the length of the longest uninterrupted CAG repeat stretch has been correlated with age at SCA1 onset. We detected CAT interruption(s) in the archived samples of Korean SCA1 patients using a traditional restriction enzyme method and validated the usefulness of a fluorescence-based tethering PCR procedure. Among the 2,312 alleles analyzed from 1,156 patients, we found 17 expanded alleles with $\geq 39$ repeats, $71 \%$ of which harbored $39-44$ repeats. Restriction enzyme method of six samples (four with 39-44 repeats and two with $>44$ repeats) revealed that none of the expanded alleles had CAT interruption(s). Tethering PCR showed the characteristic electropherogram pattern expected without CAT interruption(s). Along with the enzyme restriction method, tethering PCR can be applied to determine the number of allele repeats and provide information on CAT interruption(s) in clinical laboratories.

Key Words: Spinocerebellar ataxia type 1, CAT interruption, Tethering PCR
Received: January 3, 2021

Revision received: February 9, 2021

Accepted: September 17, 2021

\section{Corresponding author:}

Ja-Hyun Jang, M.D., Ph.D.

Department of Laboratory Medicine and

Genetics, Samsung Medical Center, 81 Irwon-ro, Gangnam-gu, Seoul 06351,

Korea

Tel: $+82-2-3410-1890$

Fax: +82-2-3410-0074

E-mail: jahyun.jang@gmail.com

\section{(1) (\$)}

C) Korean Society for Laboratory Medicine This is an Open Access article distributed under the terms of the Creative Commons Attribution Non-Commercial License (https://creativecommons.org/licenses/by-nc/4.0) which permits unrestricted non-commercial use, distribution, and reproduction in any medium, provided the original work is properly cited.
Spinocerebellar ataxia type 1 (SCA1) is an autosomal dominant disease characterized by progressive cerebellar ataxia, dysarthria, and, eventually, deterioration of bulbar functions [1]. The prevalence of SCA1 is approximately $1-2 / 100,000$, and SCA1 accounts for approximately $6 \%$ of patients with autosomal dominant cerebellar ataxia worldwide, although the proportions vary with population [2]. SCA1 is caused by an abnormal CAG repeat expansion in the ataxin 1 gene (ATXN1). Normal alleles have a repeat number ranging from 6-44, with 1-3 CAT interruption(s); approximately $78 \%$ of normal ATXN1 alleles have a configuration of (CAG)nCATCAGCAT(CAG)n, 11\% have (CAG)nCAT(CAG) n, and $4 \%$ have (CAG)nCATCAGCATCAGCAT(CAG)n [3]. Since there is an overlapping CAG repeat range (36-44 repeats) in healthy individuals and SCA1 patients, the presence or absence of CAT interruption(s) is important for interpreting the allele status within that range: CAT interruption indicates a normal allele, whereas in the case of no interruption, the allele within the 3638 repeat range is interpreted as mutable normal, and the allele within the 39-44 repeat range is interpreted as full mutation [4]. The CAT interruption status might also be a relevant indicator for patients with a full mutation allele with $>44$ repeats, as the length of the longest uninterrupted CAG repeat stretch is corre- 
lated with age on SCA1 onset [5-7].

As PCR with flanking primers followed by fluorescent fragment length analysis cannot determine the presence or absence of an interrupted sequence, PCR with restriction enzyme digestion has been used for CAT interruption detection in ATXN1 alleles [3]. Repeat-primed (RP)-PCR and tethering PCR can also recognize interruption(s) based on a characteristic electropherogram pattern [8-10]. Despite some reports on Korean SCA1 patients [11-13], little is known about the CAT interruption status of expanded alleles and the proportion of 39-44 repeat alleles among expanded alleles. As SCA1 is rare and the probability of encountering expanded alleles is very low, it is difficult to implement a routine diagnostic testing for CAT interruption(s). We compared the traditional restriction enzyme method and fluorescence-based tethering PCR approach to detect CAT interruptions in archived SCA1 patient samples to develop a new strategy combining these two methods as a diagnostic test in Korean patients. The Institutional Review Board of Samsung Medical Center, Seoul, Korea approved this study and waived the need for informed consent (IRB No. 2020-12-003).

In total, 1,156 patients were tested for SCA1 as part of the differential diagnosis of autosomal dominant spinocerebellar ataxia at the Samsung Medical Center between January 2010 and December 2019 using fluorescent fragment analysis with flanking primers [5' carboxyfluorescein (FAM)-CCAGACGCCGGGACACA-3' and 5'-CCGGAGCCCTGCTGAGGT-3'] [4]. Among the 2,312 alleles, 12 alleles (0.5\%) harbored 39-44 repeats, and five alleles $(0.2 \%)$ harbored $>44$ repeats (Fig. 1 ).

We performed SfaNI restriction enzyme digestion analysis of DNA samples from one healthy subject, three proficiency test materials from Korean Institute of Genetic Testing Evaluation, and six patients with $\geq 39$ repeats archived as positive controls

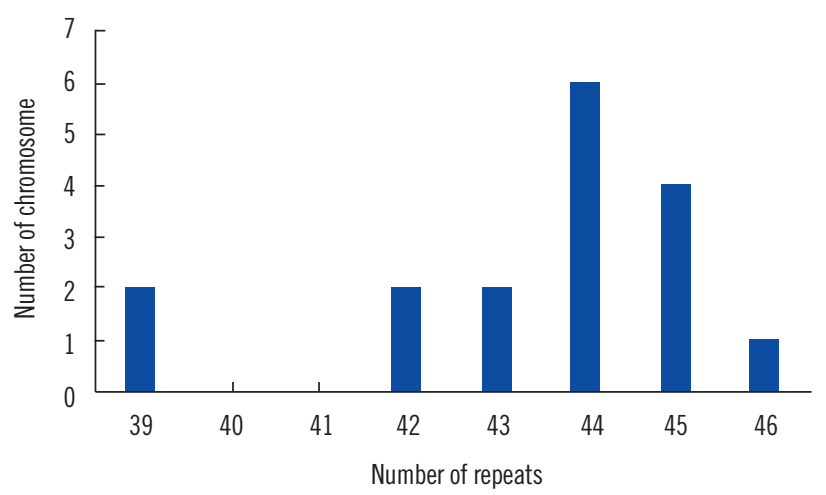

Fig. 1. Distribution of the number of repeats of ATXN1 alleles with $\geq 39$ repeats. and stored at $-20^{\circ} \mathrm{C}$ within two years from the start of the study. The number of repeats in the expanded allele varied across among patient samples: 42 (one sample), 43 (two samples), 44 (one sample), 45 (one sample), and 46 (one sample). PCR with primers 5'-AACTGGAAATGTGGACGTAC-3' and 5'-CAACATGGGCAGTCTGAG-3' followed by SfaNI enzyme digestion was performed as previously described [5]. As the SfaNI enzyme recognizes and cleaves the CAT sequence, the normal allele is expected to be cleaved, whereas the full-penetrance allele is not. Expanded alleles in the patient and proficiency test samples harbored no CAT interruption(s), whereas normal alleles did (Fig. 2), which indicated that the expanded alleles were full-penetrance alleles.

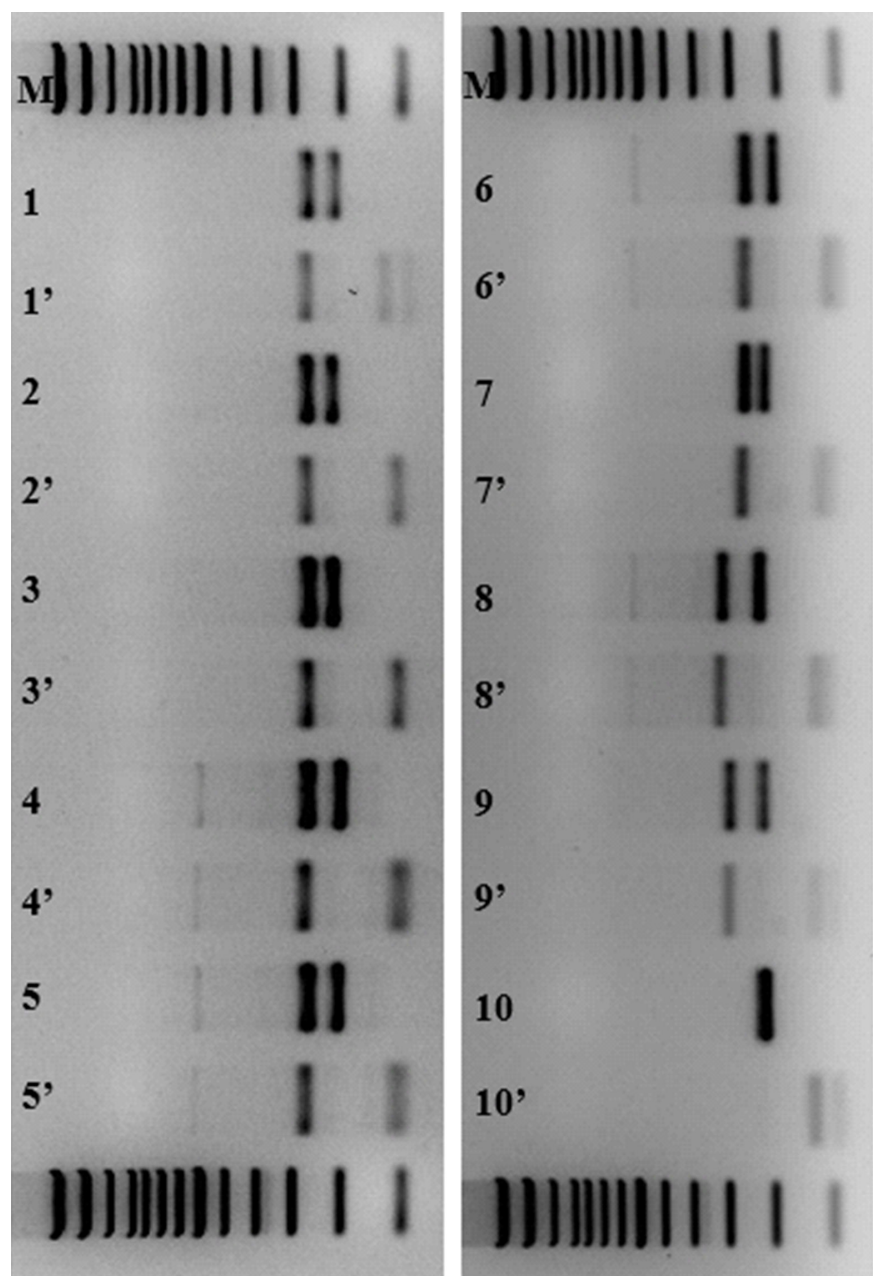

Fig. 2. Electrophoresis of PCR products before and after digestion with SfaNI enzyme. Lane M, size marker; lanes 1-6, patient samples with 43, 45, 44, 46, 43, and 42 repeats in the expanded allele, respectively; lanes 7-9, proficiency test materials with 43,60 , and 52 repeats in the expanded allele, respectively; lane 10, negative control. Lanes labeled $\mathrm{N}$ and $\mathrm{N}$ ' indicate PCR products before and after enzyme digestion, respectively. 
Tethering PCR was performed on 10 DNA samples that were tested for SfaNI restriction enzyme digestion analysis, using a locus-specific fluorescently labeled forward primer (5'-FAM-TTTGCTGGAGGCCTATTCCACTCT-3') and reverse primer (5'-GAGCCCTGCTGAGGTGCTGCTGCTGCTGCTG-3'), as previously described [10]. The reverse primer contained 16 bases of a locusspecific sequence, followed by five CTG units complementary to the (CAG)n repeats. Amplicons were loaded on an $A B I$ Prism 3730XL system (Thermo Fisher Scientific, Foster City, CA, USA) with the GS-500 size standard. Data were processed using Genemarker software version 1.95 (SoftGenetics, State College, PA, USA). Besides the main peaks caused by annealing of the reverse primer to perfectly matched bases, a stutter pattern with a 3-bp interval was obtained due to random annealing of the reverse primer within CAG repeats via the five CTG units. The first stutter peak indicated a five-CAG repeat, and the number of repeats were estimated by counting the following peaks. The presence of CAT interruption(s) was ere recognized by the loss of or reduction in signal intensity, as the reverse primer cannot bind to interrupted CAT sequences. All nine expanded alleles among the 20 alleles from the 10 DNA samples showed the expected pattern. Fig. 3 shows an electropherogram of CAT interruption patterns for representative samples. For normal homozygous samples, there was an area without a fluorescence signal due to an interrupted sequence, which the reverse primer containing the CAG repeat cannot bind to. In SCA1 patient samples with an uninterrupted expanded allele and a normal interrupted allele, there is an area where the peak height abruptly decreases.

The proportion of alleles with $\geq 39$ repeats was similar $(0.862 \%)$ to that reported in a previous UK study that analyzed 6,378 SCA1 alleles [14]; however, the proportion of the 39-44 repeat alleles among expanded alleles was higher in the present study (72\% vs. 36\%). SfaNI restriction enzyme digestion confirmed that all tested patient samples with expanded alleles had uninterrupted CAG repeats. Among the four patients with 39-44 repeat alleles, which were confirmed to have uninterrupted $C A G$ stretches, clinical information was available for three patients, and their age at disease onset ranged from 44 to 59 years. Onset in these patients seemed to be rather late, considering that the reported mean age of SCA1 onset is in the fourth decade of
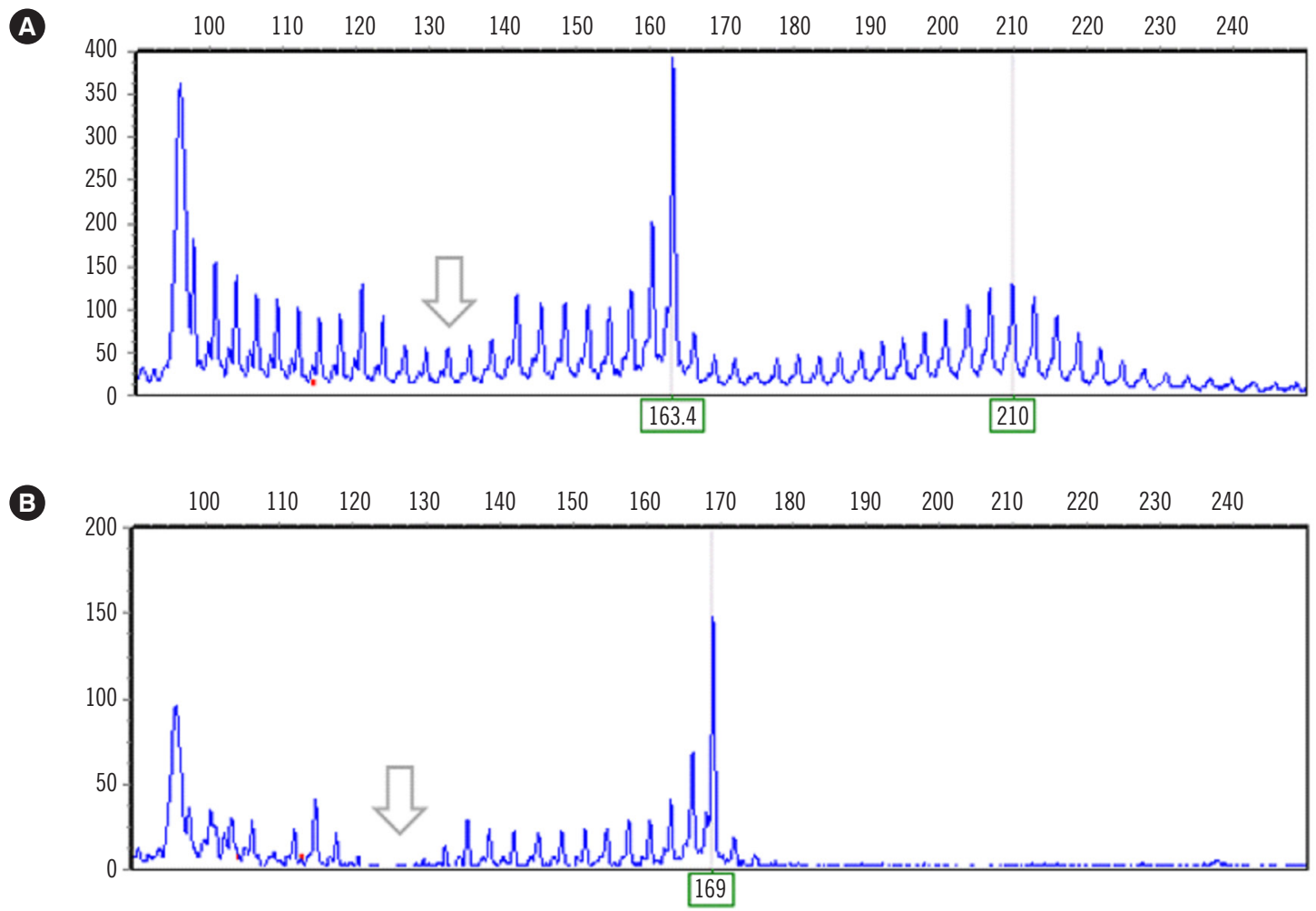

Fig. 3. Electropherograms of fluorescent fragment length analysis. (A) Electropherogram pattern of a sample with a normal interrupted (26 repeats) and an expanded uninterrupted allele (42 repeats). (B) Electropherogram pattern of a sample with a normal homozygous allele (28 repeats) with CAT interruption(s) in both alleles. The arrow indicates a reduction in signal due to the presence of interruption(s) in the normal allele. 
life $[15,16]$. However, our results are comparable to a previous report on intermediate alleles, where the mean age of SCA1 onset among patients with uninterrupted 39-44 repeat alleles was 62 years (range: 49-85 years) [17].

The present study confirms the lack of CAT interruption(s) in ATXN1 in Korean SCA1 patients. However, follow-up studies are needed since these results are based on a small sample size. Tethering PCR may be a convenient method for clinical laboratories as it allows determining the size of alleles and identifying the presence or absence of CAT interruption(s) based on a loss or reduction in fluorescence intensity. However, it does not reveal whether or not the interruption(s) is(are) present in the expanded allele. If a stutter pattern indicates the presence of interruption(s) in one allele, further restriction enzyme tests are needed to determine whether the interruption(s) is(are) present in the normal or expanded allele.

\section{ACKNOWLEDGMENTS}

None.

\section{AUTHOR CONTRIBUTIONS}

Jang JH contributed to the conception and design of the study and drafted the manuscript; Yoon SJ reviewed the data; Kim SK performed the tests; Cho JW was involved in clinical evaluation; and Kim JW supervised the study. All authors read and approved the final manuscript.

\section{CONFLICTS OF INTEREST}

None declared.

\section{RESEARCH FUNDING}

None declared.

\section{ORCID}

\author{
Ja-Hyun Jang \\ Sun Joo Yoon \\ Jin Whan Cho \\ Jong-Won Kim \\ Sun-Kyung Kim
}

\section{REFERENCES}

1. Opal P, Ashizawa T. Spinocerebellar Ataxia Type 1. 1998 Oct 1 [Updated 2017 Jun 22]. In: Adam MP, Ardinger HH, Pagon RA, et al., editors. GeneReviews ${ }^{\circledR}$ [Internet]. Seattle (WA): University of Washington, Seattle; 1993-2021. Available from: https://www.ncbi.nIm.nih.gov/books/ NBK1184/.

2. Schöls L, Bauer P, Schmidt T, Schulte T, Riess O. Autosomal dominant cerebellar ataxias: clinical features, genetics, and pathogenesis. Lancet Neurol 2004;3:291-304.

3. Chung MY, Ranum LP, Duvick LA, Servadio A, Zoghbi HY, Orr HT. Evidence for a mechanism predisposing to intergenerational CAG repeat instability in spinocerebellar ataxia type I. Nat Genet 1993;5:254-8.

4. Sequeiros J, Seneca S, Martindale J. Consensus and controversies in best practices for molecular genetic testing of spinocerebellar ataxias. Eur J Hum Genet 2010;18:1188-95.

5. Matsuyama Z, Izumi Y, Kameyama M, Kawakami H, Nakamura S. The effect of CAT trinucleotide interruptions on the age at onset of spinocerebellar ataxia type 1 (SCA1). J Med Genet 1999;36:546-8.

6. Kraus-Perrotta $C$ and Lagalwar $S$. Expansion, mosaicism and interruption: mechanisms of the CAG repeat mutation in spinocerebellar ataxia type 1. Cerebellum Ataxias 2016;3:20.

7. Menon RP, Nethisinghe S, Faggiano S, Vannocci T, Rezaei H, Pemble S, et al. The role of interruptions in polyQ in the pathology of SCA1. PLoS Genet 2013;9:e1003648.

8. Warner JP, Barron LH, Goudie D, Kelly K, Dow D, Fitzpatrick DR, et al. A general method for the detection of large CAG repeat expansions by fluorescent PCR. J Med Genet 1996;33:1022-6.

9. Yrigollen CM, Durbin-Johnson B, Gane L, Nelson DL, Hagerman R, Hagerman PJ, et al. AGG interruptions within the maternal FMR1 gene reduce the risk of offspring with fragile X syndrome. Genet Med 2012;14: 729-36.

10. Cagnoli C, Brussino A, Mancini C, Ferrone M, Orsi L, Salmin P, et al. Spinocerebellar ataxia tethering PCR: A rapid genetic test for the diagnosis of spinocerebellar ataxia types 1, 2, 3, 6, and 7 by PCR and capillary electrophoresis. J Mol Diagn 2018;20:289-97.

11. Kim JY, Park SS, Joo SI, Kim JM, Jeon BS. Molecular analysis of spinocerebellar ataxias in Koreans: frequencies and reference ranges of SCA1, SCA2, SCA3, SCA6, and SCA7. Mol Cells 2001;12:336-41.

12. Lee WY, Jin DK, Oh MR, Lee JE, Song SM, Lee EA, et al. Frequency analysis and clinical characterization of spinocerebellar ataxia types 1,2 , 3, 6, and 7 in Korean patients. Arch Neurol 2003;60:858-63.

13. Kim JS, Kwon S, Ki CS, Youn J, Cho JW. The etiologies of chronic progressive cerebellar ataxia in a Korean population. J Clin Neurol 2018; 14:374-80.

14. Nethisinghe S, Pigazzini ML, Pemble S, Sweeney MG, Labrum R, Manso $\mathrm{K}$, et al. PolyQ tract toxicity in SCA1 is length dependent in the absence of CAG repeat interruption. Front Cell Neurosci 2018;12:200.

15. van de Warrenburg BP, Hendriks H, Dürr A, van Zuijlen MC, Stevanin G, Camuzat $A$, et al. Age at onset variance analysis in spinocerebellar ataxias: a study in a Dutch-French cohort. Ann Neurol 2005;57:505-12.

16. Tezenas du Montcel S, Durr A, Rakowicz M, Nanetti L, Charles P, Sulek A, et al. Prediction of the age at onset in spinocerebellar ataxia type 1, 2, 3 and 6. J Med Genet 2014;51:479-86.

17. Zühlke C, Dalski A, Hellenbroich Y, Bubel S, Schwinger E, Bürk K. Spinocerebellar ataxia type 1 (SCA1): phenotype-genotype correlation studies in intermediate alleles. Eur J Hum Genet 2002;10:204-9. 